\title{
An Introduction to Hybrid Platform Mobile Application Development
}

\author{
Anmol Khandeparkar \\ Department of Computer \\ Engineering, \\ Mukesh Patel School of \\ Technology, Management and \\ Engineering, \\ Mumbai , India.
}

\author{
Rashmi Gupta \\ Department of Computer \\ Engineering, \\ Mukesh Patel School of \\ Technology, Management and \\ Engineering, \\ Mumbai , India.
}

\author{
B.Sindhya \\ Department of Computer \\ Engineering, \\ Mukesh Patel School of \\ Technology, Management and \\ Engineering, \\ Mumbai , India.
}

\begin{abstract}
The mobile applications development industry has been developing at a rapid pace. The various operating systems available in the market are diverse and this proves to be a hindrance to application developers while developing a single application for all the operating systems. Hybrid platform mobile applications help in cost cutting and saving time as well as providing components for easier development of applications which provide a native feel to the user. This paper aims to help developers make the right choice in order to build an application as well as give vital information about hybrid platform mobile application approaches and their advantages and disadvantages.
\end{abstract}

\section{Keywords}

Hybrid platform mobile applications, frameworks, native approach, interface.

\section{INTRODUCTION}

Native applications are developed using the native programming languages of the devices for which it needs to be developed. For example, The native programming language for iOS is objective $\mathrm{C}$ and the new swift and for android, applications are built using JAVA programming language[1].

Native apps provide with the best usability, features and best overall user experience. The characteristic that any native application would possess are:

1. Multi touch: allows the user to double tap, pinch-spread and other complex UI gestures.

2. Fast graphic API: any native application provides with the fastest graphics depending on the inbuilt characteristics of various devices.

3. Fluid animation: is very essential for providing a good gaming experience on the device. It is also necessary for highly interactive reporting or compound computational algorithms for transforming audio and videos.

4. Built-in components: inbuilt application such as camera, address book, photo gallery, geolocation and other features can provide their services to mobile apps

5. Ease of use: the user interface of native applications is easily understandable by users which enables them to seamlessly interact with the application [2].
Applications have rapidly caught up in recent times ever since app stores were made for the mobile phones developers have become more and more interested in creating apps for different operating system and platforms. But the problem was that the same application had to be created for different operating systems which led to wastage of resources and time. Therefore, Hybrid platforms for coding using Html, CSS and JavaScript were created which led to a new beginning of mobile applications. Hybrid platform apps are developed using technologies such as: HTML, CSS and JavaScript. It is then placed into a native container such as Adobe PhoneGap. These native containers run the application code and package it into an app. Therefore it provides us with various Mobile Application Development Platforms that can generate largely native apps from a single original codebase, which can be deployed across various mobile platforms.

\section{APPROACH TO BUILD A HYBRID PLATFORM MOBILE APPLICATION}

The Hybrid Approach may be seen as a bridge between Web and Native approaches. A Hybrid application is built using $\mathrm{Html}$, CSS and JavaScript which are web technologies and are executed in the native Hybrid approach uses the browser engine of the device which renders and displays the HTML content in full screen Web view control. Even during the development of Hybrid applications on the desktop, we can view the application in the browser. The device capabilities are exposed to the hybrid application through an abstraction layer. The abstraction layer exposes the device capabilities as JavaScript Application Programming Interface. Hybrid approach can take the advantage of both browser engine and device capabilities. Hybrid approach can be used for both server backed and standalone applications. Unlike web applications Hybrid applications needs to be downloaded and installed on the mobile device [3].

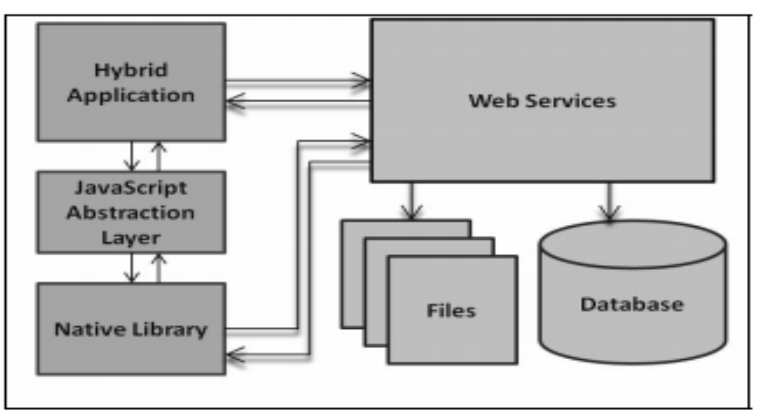

Figure 1: Hybrid Application 


\section{FRAMEWORKS FOR DEVELOPING HYBRID PLATFORM MOBILE APPLICATIONS}

Various different frameworks go into building a hybrid platform mobile application. It is necessary to select the framework in order to create a hybrid platform application which has great performance along with an easy to use and a beautiful user interface. Various frameworks are available in the market for developing hybrid platform mobile applications. A comparison of the frameworks available in the market is done to ensure that the developer makes the appropriate choice of framework. The table below rates the frameworks on a scale of 1-10 on the basis of documentation provided by the framework, performance of the framework, the active community support of the framework and the learning curve.

\begin{tabular}{|l|l|l|l|l|}
\hline $\begin{array}{l}\text { Framewor } \\
\mathbf{k}\end{array}$ & $\begin{array}{l}\text { Document } \\
\text { ation }\end{array}$ & $\begin{array}{l}\text { Perform } \\
\text { ance }\end{array}$ & $\begin{array}{l}\text { Commu } \\
\text { nity }\end{array}$ & $\begin{array}{l}\text { Learn } \\
\text { ing } \\
\text { Curve }\end{array}$ \\
\hline Ionic & 8 & 5 & 7 & 7 \\
\hline $\begin{array}{l}\text { Famo.us/An } \\
\text { gular }\end{array}$ & 6 & 10 & 4 & 4 \\
\hline OnSenUI & 7 & 5 & 3 & 9 \\
\hline
\end{tabular}

Table 1. Comparison of different frameworks

\section{COMPARISION BETWEEN HYBRID AND NATIVE APPROACH}

There are various pros and cons in using native or hybrid application development technology. In this section we are going to discuss about the various advantage and disadvantage of using hybrid app development:

\section{Design of Interface:}

Mobile interfaces can be designed using both native and hybrid technologies. However, it is more flexible in designing interfaces using hybrid platform technologies such as HTML5 and CSS3. But there are certain design issues when it comes to designing interfaces with hybrid platform technologies such as it is tedious and time consuming for implementing complicated application interfaces.

Whereas, it is much easier to create interfaces using native platform technologies as it provides with different tools and design widgets to create standard interfaces which are not available with hybrid platform tools.

\section{Cost:}

It is more cost effective to develop applications using hybrid mobile technologies because you can build an application once and submit it to different platforms(iPhone, Windows, Android etc) using PhoneGap which would save a lot of money by not having to build an application in different programming languages supported by the different platforms such as the Android or iOS.

Whereas, developing an application using native platform technologies can be more costly than developing from a hybrid platform technology.

\begin{tabular}{|l||l||l|}
\hline Description & $\begin{array}{l}\text { Small to Medium Size Projects } \\
\text { Average Cost } \\
\text { Native App Development }\end{array}$ & $\begin{array}{l}\text { Small to Medium Size Projects } \\
\text { Average Cost } \\
\text { Hybrid App Development }\end{array}$ \\
\hline \hline $\begin{array}{l}\text { Back-end programming, APIs, } \\
\text { admin and cloud deployment }\end{array}$ & $\$ 10,000-\$ 20,000$ & $\$ 10,000-\$ 20,000$ \\
\hline \hline iOS native development & $\$ 15,000-\$ 30,000$ & $\$ 0$ \\
\hline \hline Android native development & $\$ 10,000-\$ 20,000$ & $\$ 0$ \\
\hline \hline $\begin{array}{l}\text { Hybrid app development using } \\
\text { PhoneGap technology }\end{array}$ & $\$ 0$ & $\$ 10,000-\$ 20,000$ \\
\hline \hline Total & $\$ 35,000-\$ 70,000$ & $\$ 20,000-\$ 40,000$ \\
\hline
\end{tabular}

Figure 2: Average cost of mobile app development using hybrid vs native mobile app development.

\section{Development Timeline:}

Hybrid applications are usually faster and easier to develop and implement. However, if they have many complicated native features that are beyond the capability of the hybrid application Framework and Environment then it will be more time consuming to develop such an application.

\section{User Experience and Performance:}

Native applications tend to provide better performance, responsive and fluid experience to its user without any delays than hybrid platform applications. Here are some examples of user interaction experience of native and hybrid platform application:

Clicks are more responsive in the case of native applications but with hybrid the user might have to click more than once to get a particular response from the application which at times leads to frustrated and unsatisfied users.

Scrolling in a native application is seamless but in the case of hybrid application the user might feel delay in loading frames in the sequence.

Large animation in a native application can be executed with great fluidity but in case of hybrid application large animation may not behave seamlessly.

\section{Maintainability:}

Hybrid platform application is more maintainable as long as the developer selects the correct framework such as kendoUI, JQuery mobile, ionic, etc. and codes it properly because it is easier to maintain web app technology like HTML, CSS, and JavaScript than a native platform application.

\section{Security:}

Depending on the security requirements of the project, native apps may provide better security environment however, most of the security issues are created based on developer's lack of knowledge and certain server side security issues.

Whereas, if a hybrid platform application requires many security features then these features needs to be implemented in a hybrid platform environment.

\section{Platform independent:}

One of the major advantage of developing an application in the hybrid platform environment is that the application needs to be coded only once and can be deployed across various other platforms which is not possible in the case of native platform application. 


\section{Tools and Debugging:}

Native development environment provides better tools for testing and debugging the tools so that it is easier and less time consuming in finding the bug and fixing it. Whereas, for a hybrid platform there are not many tools available for testing and debugging purpose because of which the developer ends up spending additional hours in finding and fixing the bug [1].

\section{CONCLUSION}

The field of mobile application development has developed rapidly in the last few years. Native mobile applications were the only type of applications which were common in the past. Native mobile applications without doubt provide the best user experience however, hybrid platform mobile applications will be preferred when the application is to be made for multiple platforms and time and cost are the primary factors.
A developer needs to weigh the pros and cons of both hybrid and native applications and choose the right approach to building an application based on experience and the application's purpose.

\section{REFERENCES}

[1] Bernard Kohan and Joseph Montanez, "A comparison of native app development (iPhone: Objective-C / Swift, Android: Java) vs hybrid / PhoneGap app development (HTML5, CSS, JavaScript)".

[2] Mario Korf and Eugene Oksman, "Native, HTML5, or Hybrid: Understanding Your Mobile Application Development Options".

[3] Rahul Raj C.P and Seshu Babu Tolety, "A study on approaches to build cross-platform mobile applications and criteria to select appropriate approach" 